Int. J. Dev. Biol. 63: 105-112 (2019)

https://doi.org/10.1387/ijdb.180329jf

\title{
Function of atypical mammalian oocyte/zygote nucleoli and its implications for reproductive biology and medicine
}

\author{
JOSEF FULKA, Jr. ${ }^{*}, 1$, MICHAL BENC ${ }^{1}$, PASOUALINO LOI ${ }^{2}$, ALENA LANGEROVA ${ }^{3}$ and HELENA FULKA ${ }^{1,4}$ \\ ${ }^{1}$ Institute of Animal Science, Prague, Czech Republic, ${ }^{2}$ University of Teramo, Teramo, Italy, \\ ${ }^{3}$ GENNET, Prague, Czech Republic and ${ }^{4}$ Institute of Molecular Medicine, Prague, Czech Republic
}

\begin{abstract}
Mammalian oocytes/zygotes contain atypical nucleoli that are composed exclusively of a dense fibrillar material. It has been commonly accepted that these nucleoli serve as a repository of components that are used later on, as the embryo develops, for the construction of typical tripartite nucleoli. Indeed, when nucleoli were removed from immature oocytes (enucleolation) and these oocytes were then matured, fertilized or parthenogenetically activated, development of the produced embryos ceased after one or two cleavages with no detectable nucleoli in nuclei. This indicated that zygotic nucleoli originate exclusively from oocytes, i.e. are maternally inherited. Recently published results, however, do not support this developmental biology dogma and demonstrate that maternal nucleoli in one-cell stage embryos are necessary only during a very short time period after fertilization when they serve as a major heterochromatin organizing structures. Nevertheless, it still remains to be determined, which other functions/roles the atypical oocyte/ zygote nucleoli eventually have.
\end{abstract}

KEY WORDS: nucleolus, oocyte, zygote

\section{Introduction}

Mammalian oocytes that are reaching or reached their full size, zygotes and early cleavage embryos contain atypical nucleoli (Hyttel, 2011). In somatic cells, as well as in more advanced embryonic cells (including embryonic stem cells), nucleoli contain three distinct compartments - the fibrillar, dense fibrillar and granular components (Jordan, 1991). These differentiated nucleoli are engaged in many cell functions, i.e. rDNA transcription and ribosome production, cell cycle progression, differentiation, aging and others. For this reason, nucleoli are commonly viewed as multifunctional intracellular organelles (Boisvert et al., 2007). Nucleoli in growing oocytes have the same morphology as fully differentiated nucleoli, but in parallel with the oocyte growth, the nucleolar morphology gradually changes and finally, after oocytes reach the end of this growth period and attain their full size, the nuclei (germinal vesicles - GVs) in the most developmentally advanced oocytes contain only round dense structures that are enclosed with a ring of chromatin (heterochromatin) (Bonnet-Garnier et al., 2012). In parallel with this transformation, transcription gradually ceases (Bogolyubov, 2018; Crozet, 1989). These atypical dense nucleoli found in fully grown oocytes are commonly called as nucleolus like bodies (NLBs).
It must be noted here, that in fully grown oocytes two distinct patterns of chromatin organization in GVs exist. In the most advanced oocytes, NLBs are enclosed with heterochromatin (SN-surrounded nucleolus), whilst in less advanced oocytes chromatin is dispersed in GVs (NSN - non surrounded nucleolus). After fertilization (or parthenogenetic activation), embryos from SN oocytes develop at least up to blastocysts. On the other hand, embryos originating from the NSN oocytes cleave only to the two-cell stage and then their development is arrested (Zuccotti et al., 2011).

Interestingly, after fertilization or parthenogenetic activation morphologically similar intranuclear organelles can be seen in pronuclei (PNs), but as embryos develop further, these structures are gradually transformed into typical tripartite nucleoli. Concomitantly with this transformation, rRNA production is initiated again (Fulka and Aoki, 2016). It has been commonly accepted that nucleoli (NPBs) in zygotes serve as a repository of material(s) from which, as the embryo develops, typical tripartite nucleoli with relevant functions

\footnotetext{
Abbreviations used in this paper: GV, germinal vesicle; GVBD, germinal vesicle breakdown; ICSI, intracytoplasmic sperm injection; IVF, in vitro fertilization; MI, metaphase I; MII, metaphase II; NLB, nucleolus like body; NPB, nucleolus precursor body; PN, pronucleus.
}

*Address correspondence to: Josef Fulka, Jr. Institute of Animal Science, Pratelstvi 815, 10400 Prague 10, Czech Republic.
Tel: +420 267009 606. E-mail: fulka @ vuzv.cz

Submitted: 2 October, 2018. Accepted: 11 October, 2018.

ISSN: Online 1696-3547, Print 0214-6282 
are formed. For this reason, the nucleoli in zygotes are referred to as "nucleolus precursor bodies (NPBs).

Nevertheless, some observations indicated that the role of NPBs may be more complex. For example, it has been reported that the distribution and number of NPBs in pronuclei can serve as an indicator of developmental potential in human one cell stage embryos. The zygotes with an equal (or very similar) number and distribution of NPBs in both pronuclei had much higher developmental potential when compared to those zygotes where these parameters were skewed (Tesarik and Greco, 1999). The explanation for this phenomenon is very difficult to find, especially if we accept the idea of NPBs as a passive nucleolar material storage site. Nucleoli as well as NLBs/NPBs are dissolved in the cytoplasm with the onset of every cell division and when nuclei are later formed again, the nucleolar material reappears in newly assembled nuclei. Therefore, in principle the embryonic nuclei (including pronuclei) should always contain equal or similar quantities of the "stored" nucleolar material (Fulka et al., 2015). The nucleolar material distribution should be further equalized during the next embryonic cleavage, i.e. to the two cell stage. Finally, why should the NPB material distribution matter during an embryonic phase where there is no detectable rRNA production (zygotes in mice; up to the four cell stage in humans)? Could NLBs/NPBs have other functions unrelated to rRNA production?

\section{New era in NLB/NPB research}

A new era in oocyte/zygote nucleolus research began in 2003, when two seminal papers were published. First, Burns et al. (2003) produced Npm2 knockout mice. NPM2 (nucleoplasmin 2) is essential for sperm head chromatin decondensation in amphibians. This, however, is not true for the mouse (Burns et al., 2003; Inoue et al., 2011; Ogushi and Saitou, 2010). The oocytes (GVs) and consequently zygotes (PNs/pronuclei) from Npm2 knockout mice have normally sized pronuclei which lack nucleoli (NLBs/ NPBs) and their development is compromised. Interestingly, in very exceptional cases the authors obtained blastocysts and few offspring. This led them to postulate the existence of some compensatory mechanisms allowing the nucleoli-less embryos to develop to term. Moreover, it must be also mentioned here, that oocytes from these knockout mice matured normally in vitro with the same kinetics as control oocytes and without apparent chromosomal separation abnormalities.

Second, in the same year, Fulka, Jr. et al. (2003) reported that NLBs can be removed relatively simply from GVs of immature porcine oocytes (enucleolation). The enucleolated oocytes matured to metaphase II with the same frequency as controls. This indicated that NLBs are not essential for the onset of meiosis (GVBD - germinal vesicle breakdown) and the progress of oocyte maturation. This was somehow surprising, because nucleoli in somatic cells and yeasts play an important role especially during the exit from metaphase to anaphase (Bachant and Elledge, 1999). That NLBs can be removed from oocytes of some other species (assuming they are visible in GVs - in this case the mouse; Fig. 1 for demonstration) has been reported next year (Fulka et al., 2004). Moreover, this paper reported for the first time that when oocytes are enucleolated, then matured and fertilized or parthenogenetically activated, pronuclei of the resultant embryos do not contain visible nucleoli (NPBs) and the embryos without NPBs arrest after one or two cleavages. Then their development stops. This means that pronuclear NPBs are maternally inherited and once nucleoli (NLBs) are removed from GVs the nucleolar material is not newly synthetized. From the technical point of view, it must be noted here that NLBs can be also removed from human GV-stage oocytes (Langerova, unpublished; Fig 2) as well as from mononucleolar pronuclei in the mouse (Fulka, Jr. et al., 2011). Conversely, NLBs/ NPBs can also be injected into oocytes or zygotes. This allows one to add extra or substitute the missing NLB/NPB material when either intact or nucleolus-deficient oocytes or zygotes are used. The injected nucleolar material is rapidly translocated into the nucleus where very tiny NLBs/NPBs appear first, but with additional time post-injection, these tiny NLBs/NPBs fuse and form a single large nucleolus.

The reinjection of NLBs into previously enucleated oocytes can rescue their developmental potential after parthenogenetic activation or fertilization. When performed, the pronuclei con-
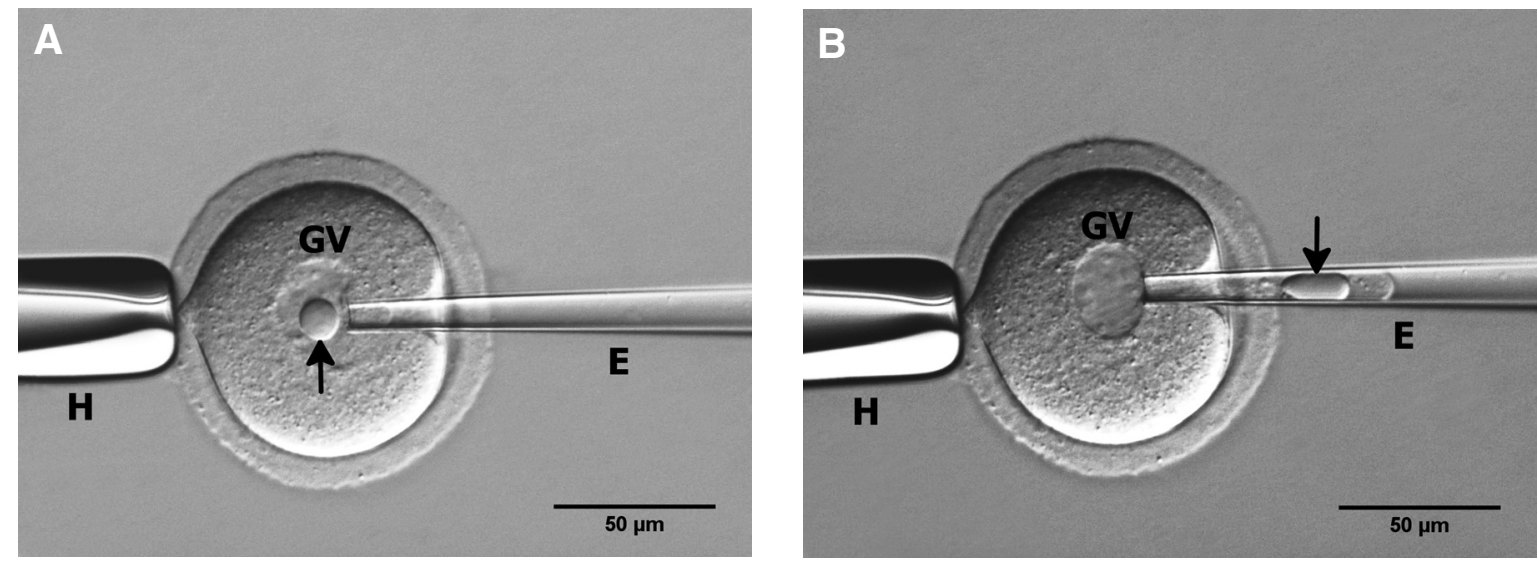

Fig. 1. Microsurgical removal of a nucleolus like body (NLB) from an immature mouse oocyte. (A). The enucleolation pipette first penetrates zona pellucida and is slowly pushed near the NLB. The pipette tip does not penetrate the vitelline or germinal vesicle membrane (GV). The oocyte diameter is approximately $80 \mathrm{\mu m}$. (B) When close contact between the tip of the enucleolation pipette and the NLB is achieved, very mild suction in the enucleolation pipette is applied and this aspirates the NLB which penetrates the GV membrane and is located in the oocyte cytoplasm (arrow). Then NLB can be detached completely from the oocyte (see Fig. 6) in the form of a so called "nucleoloplast." Note, that the GV is empty with no NLB. Abbreviations: H, holding pipette; E, enucleolation pipette; arrow, nucleolus; GV, germinal vesicle. 

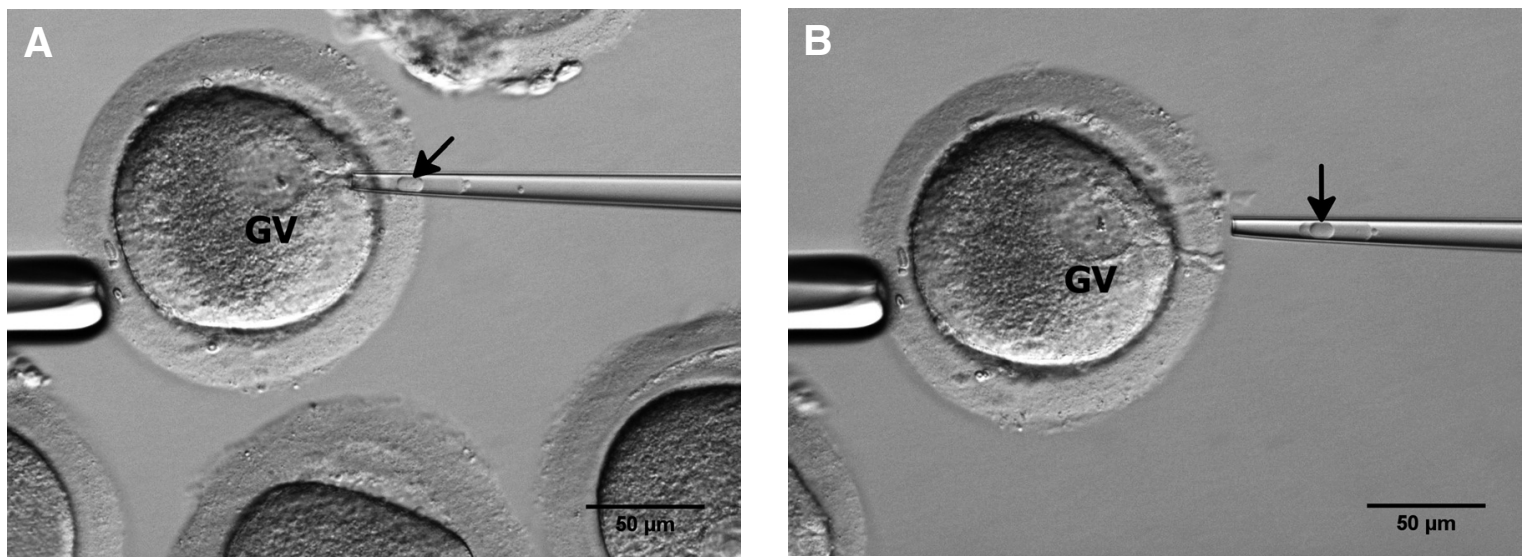

Fig. 2. Enucleolation of human immature oocytes. Enucleolation is only possible in those oocytes where NLBs are clearly visible in GVs (mouse, human, pig, etc.). (A) Here, spare human oocytes of a rather low quality were used for enucleolation. The diameter of human oocytes is approximate 120 um. (B) The nucleolus is completely removed from the oocyte as a "nucleoloplast" (arrow). The nucleoloplast is enclosed with the oocyte vitelline membrane and contains a minimum volume of the oocyte cytoplasm. Abbreviations: GV, germinal vesicle; Arrow, aspirated nucleolus in the enucleolation pipette.

tained again visible NPBs and were able to reach the blastocyst stage. Interestingly, somatic cell or even stem cell nucleoli cannot substitute for the original oocyte nucleolar material (Ogushi et al., 2008). Further characterization of enucleolated and subsequently NLB-reinjected oocytes demonstrated essentially no differences compared to intact oocytes, i.e. karyotypes, speed of maturation, spindle morphology and DNA replication post activation were not affected (Ogushi et al., 2008).

Taken together, the above mentioned results indicated that the "nucleolus material repository" theory might be indeed correct. However, without a detailed knowledge of the NLB/NPB composition other hypotheses could not be ruled out and the idea of an oocyte-specific nucleolar protein with a function unrelated to ribosome biogenesis was just as likely.

\section{Composition of NLBs/NPBs}

In contrast to differentiated nucleoli of somatic cells, where these are well characterized either by immunofluorescence or advanced proteomic analyses, the detailed characterization of NLBs/NPBs is rather complicated (Fulka and Aoki, 2016). First, when the classical immunofluorescence protocol is used, the main problem is that selected antibodies do not penetrate NLBs/NPBs, probably because of their very dense/compact structure.

The first protein convincingly demonstrated to be present in NPBs was NPM2, when mRNA encoding a GFP-tagged version was injected into immature mouse oocytes (Inoue and Aoki, 2010). Later on, with the use of an antigen retrieval approach or after treating the oocytes with proteinase $\mathrm{K}$, it has been demonstrated by independent groups that the fully grown oocyte nucleoli contain some of the typical nucleolar proteins, such as fibrillarin, B23 (nucleophosmin) and C23 (nucleolin; all involved in pre-rRNA processing), as well as RNAs, but not rRNAs. Compared to growing oocytes, labeling against UBF (upstream binding factor; RNA polymerase I transcription initiation) was rather weak (Fulka and Langerova, 2014; Shishova et al., 2014; Lavrentyeva et al., 2017). It must be noted here, that with the use of conventional immunofluoresce some other proteins were reported to be present in NLBs - i.e. LIN28, lamin A and B, TRF2, SURF6, OCT4 and others (Pochukalina et al., 2016; Romanova et al., 2006; Vogt et al., 2012). They were mostly detected on the NLB surface and it must be verified further if they are indeed bona fide NLB components as their seeming localization to NLBs might have been caused by an antibody cross-reactivity. Therefore, the exact protein composition of NLBs/NPBs remains to be determined.

When considering a detailed proteomic analysis the isolation of sufficient number of NLBs/NPBs is critical. However, assessing the minimum number of NLBs/NPBs necessary for such analysis is not an easy task. While NLBs can be easily isolated from fully grown oocytes microsurgically, they dissolve relatively rapidly when they are kept in the manipulation medium. Thus, to protect them from dissolving, NLBs are isolated from oocytes as so-called nucleoloplasts. This means that the nucleolus is enclosed with the oocyte vitelline membrane, therefore the nucleoloplast also contains a minimum volume of the oocyte cytoplasm. Before NLBs collection the nucleoloplast membrane is broken down (by piezo pulses for example) and NLBs are released into the manipulation medium. When the contact between NLBs is induced, they fuse rapidly, thus forming a giant nucleolus that can be easily handled as it is well visible. Logically, all these steps must be done very quickly. When using this approach we have found that single NLB contains approximately $1.6 \mathrm{ng}$ of proteins (Fulka et al., 2012). However, a routine proteomic analysis requires micrograms of proteins. Therefore, the number of NLBs that would be required is enormous, making the microsurgical NLB isolation impractical, but no alternative protocols has been devised to date and the exact protein composition of NLBs/NPBs remains elusive.

As the research oriented on oocyte/zygote nucleoli continued some rather puzzling and difficult to answer questions appeared.

\section{NLB/NPB puzzles}

Ogushi and Saitou (2010) studied the developmental potential of enucleolated oocytes reinjected with NLBs in more details. When enucleolated oocytes were reinjected with NLBs at the GV stage and the matured oocytes were then fertilized, up to $67 \%$ of manipulated embryos reached the blastocyst stage. When NLBs were injected into mature, previously enucleated oocytes (MII) 

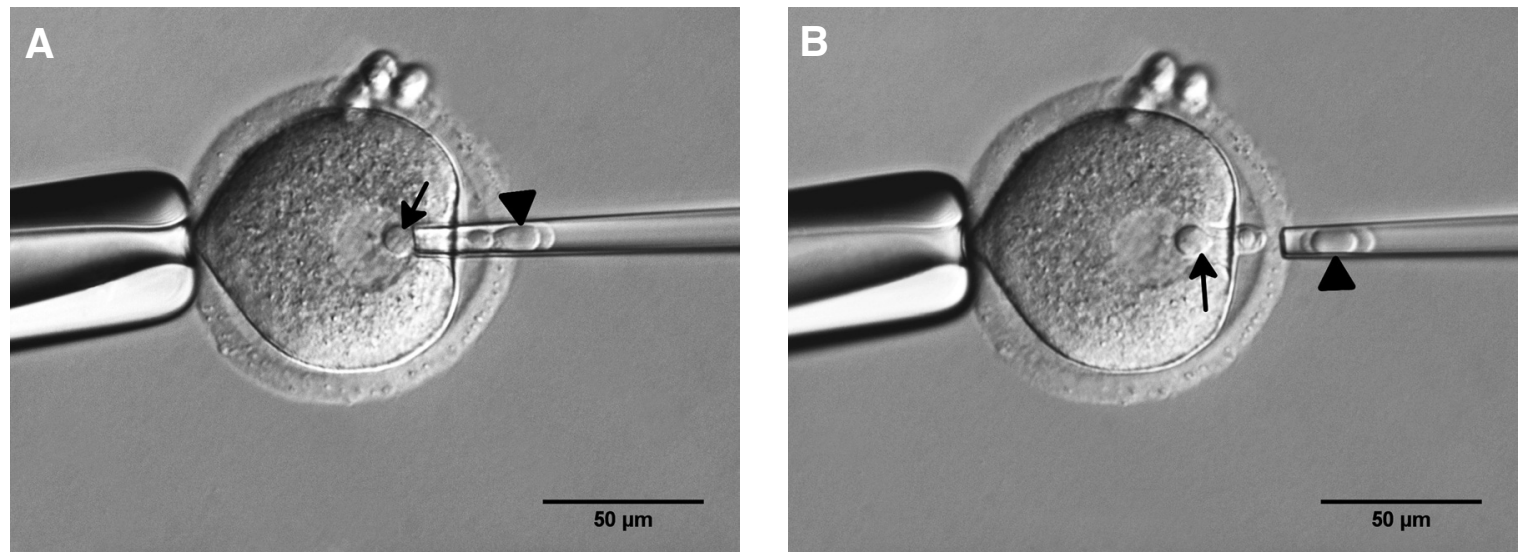

Fig. 3. Scaling the nucleolus volume to one half. (A) When the nucleolus is aspirated from the germinal vesicle (GV) and approximately one half is already outside the GV, the nucleolus can be split into two equal halves. (B) The half nucleolus can be completely removed from the oocyte. The oocytes with reduced nucleolar volume mature normally to metaphase II. Abbreviations: arrow, germinal vesicle; arrowhead, half nucleolus.

and these oocytes were then fertilized, the percentage of blastocysts obtained was lower (49\%). What was, however, much more interesting was the injection of NLBs into zygotes originating from enucleolated oocytes at 8 or $15 \mathrm{~h}$ post fertilization. Here, blastocysts were obtained only sporadically. These results strictly spoke against the originally postulated dogma that NPBs serve as a repository of material from which fully functional embryonic nucleoli are formed. If this dogma was indeed valid, it should not have mattered when the NLB material was introduced back as long as it was prior to the genome activation and ribosome production re-initiation. This step occurs at the two-cell stage in the mouse. Therefore, NPBs must have other functions in early embryogenesis.

The detailed characterization of chromatin in PNs without NPBs showed its disorganization. Whilst in control zygotes the heterochromatin encloses NPBs, in PNs with no NPBs, heterochromatin was organized typically in several clusters. Essentially, the same has also been observed in early experiments published by Burns et al. (2003). But why is the correct chromatin organization so important? It has been shown that enucleolated oocytes are apparently normal with no detectable chromosome damage or missegregation. Therefore, the NLB/NPB essential function must be restricted to early embryogenesis. This was further strengthened by experiments when MII oocytes reinjected with NLBs were fertilized by spermatozoa and live pups were obtained (Ogushi and Saitou, 2010).

The maternal and paternal chromatin is decondensed in pronuclei and if we assume, that both these sets are initially normal, then some chromatin modifications must occur during the decondensing phase and NPBs must play an essential role in these modifications. It must be noted here, that NPBs in pronuclei represent the most prominent heterochromatin organizing structures (Aguirre-Lavin et al., 2012). It is therefore conceivable, that it might be specifically heterochromatin, that is affected by the NPB absence.

\section{Towards clarifying the role of NPBs in zygotes}

The answer to some of the questions above came from experiments performed by Fulka and Langerova (2014), who analyzed early cleavage embryos originating from enucleolated oocytes in more detail. First, the authors showed that there are essentially no differences between embryos with and without NPBs (produced from enucleolated oocytes) when initial steps of ribosome biogenesis are assessed, i.e. neither RNA polymerase I activity is affected (pre-rRNA synthesis) nor is the pre-rRNA processing. The NPBs are, however, indispensable for synthesis of major and minor satellite transcripts. The NPB absence is also correlated with evident reduction of satellite DNA. At the same time, the first S-phase is abnormal and chromosomes remain connected during the first mitotic division and consequently the embryos without NPBs do not developed further.

In agreement, Kyagoku et al., (2014) enucleolated one-cell stage embryos produced by ICSI at $10 \mathrm{~h}$ post sperm injection. The authors used only those embryos where both pronuclei contained just one NPB. Surprisingly, these enucleolated zygotes developed to blastocysts and after their transfer to recipient females, offspring were born. Moreover, these authors demonstrated normal nucleologenesis in manipulated embryos (i.e. in embryos originating from enucleolated zygotes). This further supports the view, that the role of NPBs is independent of ribosome production.

Finally, Ogushi et al., (2017) demonstrated that development of zygotes originating from oocytes without NLBs can be rescued by injecting them with Npm2 mRNA. Interestingly, when $1.2 \mu \mathrm{g} / \mu \mathrm{l}$ of mRNA was injected, the pronuclei contained very small NPBs and the zygotes reached the blastocyst stage only sporadically. When mRNA concentration was increased ten times $(11 \mu \mathrm{g} / \mu \mathrm{l})$, the pronuclei contained very large NPBs with apparently normal morphology. It is not known, why reconstituted NPBs were so big, but a continuous NPM2 protein production from the exogenous mRNA is likely the cause. Surprisingly, these embryos developed to blastocysts quite well $(50 \%)$ with offspring born after their transfer to recipients.

The simplified graphical summary of NLB/NPB removal and transplantation experiments is presented in Fig. 6 .

\section{Oocyte nucleolus size scaling}

The above mentioned observations concerning the NPB size in relation to developmental potential of injected embryos are somehow surprising. It is commonly accepted, that correct nucleocytoplasmic ratio as well as the correct number and size of cell organelles are 
necessary for normal cell function (Edens etal., 2012). For example, some cancer cells have abnormal nucleocytoplasmic ratio, distinct shape of nuclei as well as some organelle abnormalities (Zink et al., 2004). This is true for mammalian oocytes and embryos. For example a low number of mitochondria or abnormal sizes of pronuclei result in poor or no development (Gardner et al., 2015; Papale et al., 2012).

We studied if this scaling also applies to mammalian oocyte NLBs. By micromanipulation, we have reduced or increased the nucleolar (NLB) material content in mouse immature oocytes and thereafter we evaluated their development after parthenogenetic activation ( $\mathrm{SrCl}_{2}$ and cytochalasin $\mathrm{B}$ - to produce diploid embryos). As mentioned above, the oocytes with no NLBs (enucleated) mature well in culture and reach metaphase II with the same frequency as controls. However, the embryos originating from these oocytes cleave just once or twice and then their development ceases. In controls, approximately $80 \%$ of sham-operated oocytes complete maturation, but by contrast, when activated, approximately $80 \%$ of them developed to the blastocyst stage. The oocytes with reduced NLB volume (Fig. 3) to $1 / 2$ mature similarly well and show the same activation rate as controls $(\sim 80 \%)$, but their development post activation is compromised and only about $30 \%$ reach the blastocyst stage. The oocytes with less than $1 / 2$ of NLBs or with some NLBs remnants do not cleave beyond the two-cell stage just like embryos from completely enucleolated oocytes, although some tiny NPBs can be seen in pronuclei. More interesting results are obtained when extra NLB material is injected into oocytes. In this case, we injected from 1 to 4 freshly isolated NLBs into immature GV stage (Fig. 4) or maturing (metaphase I) oocytes. In immature oocytes, the injected NLBs are rapidly translocated into GVs but in maturing oocytes NLBs disappear rapidly. In both these combinations, the injected oocytes mature well in culture $(\sim 80 \% \mathrm{MII})$ and the activation rate is similarly good. The pronuclei then contain much larger NPBs depending on the amount of the NLB material injected. Whilst in non-injected, two-cell stage blastomere nuclei, several nucleoli can be seen, the embryos with surplus nucleolar

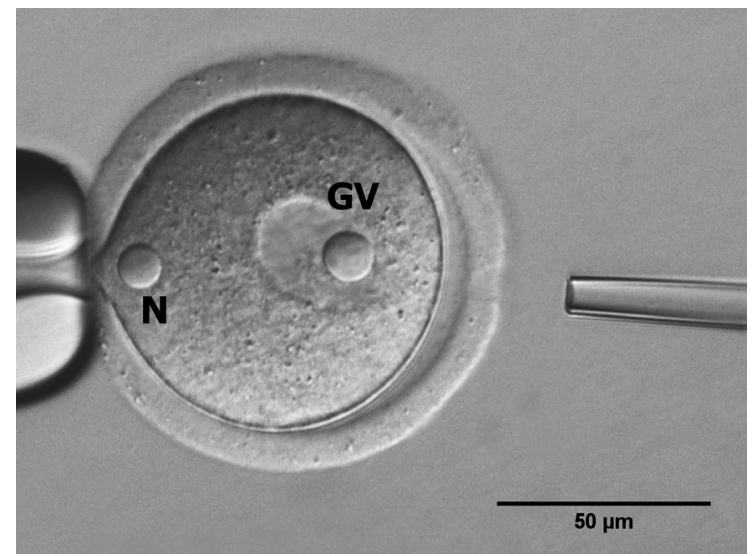

material contain just a single large nucleolus (Fig. 5). Surprisingly, the embryos with surplus nucleolar material compact earlier when compared to controls. Already at the four-cell stage the blastomere round shape disappears and blastomeres are closely apposed each to other. The embryos with 1 to 2 injected NLBs develop well to the blastocyst stage (75\%). When three NLBs are injected, 50\% embryos reach the blastocyst stage and with 4 injected NLBs only about $35 \%$ of normal blastocysts can be obtained. The reason for this decline is currently unknown. Nevertheless, when we labeled the resulting blastocysts with anti-Oct4 (Pou5f1) antibody, in all groups they contained a prominent ICM (inner cell mass) and essentially the same number of cells as controls.

Taken together, these results indicate that the reduction of NLBs material has more serious and detrimental consequences that result in compromised development. Moreover, it is also evident that the enucleolation must not be absolute. Previously we have demonstrated that typically about $5 \%$ of residual NLB material remains in GVs after enucleolation (Fulka and Langerova, 2014). Thus, the removal of about of 2/3 of NLBs has the same effect as the complete enucleolation.

Essentially, our observations confirmed the results obtained by Ogushi et al. (2017) who injected Npm2 mRNA into previously enucleolated mouse oocytes. The embryos where pronuclei contained tiny NPBs did not develop after fertilization. On the other hand, the embryos where pronucleicontained huge NPBs developed as well as controls. Similar results were also obtained by Kyogoku et al., (2018) who injected extra NLBs into MII oocytes which were then either fertilized or used as cytoplasts for SCNT. In contrast to controls, where pronuclei contained several small sized NPBs, the pronuclei in NLBs injected oocytes contained a single double sized nucleolus. The embryos in both groups developed normally with no significant developmental rate differences as well as with an equal natality post embryo transfer to recipients.

However, there are some discrepancies: In embryos originating from $\mathrm{Npm}^{+/-}$oocytes the PNs seem to contain NPBs that are smaller when compared to wild type zygotes, yet these mice were

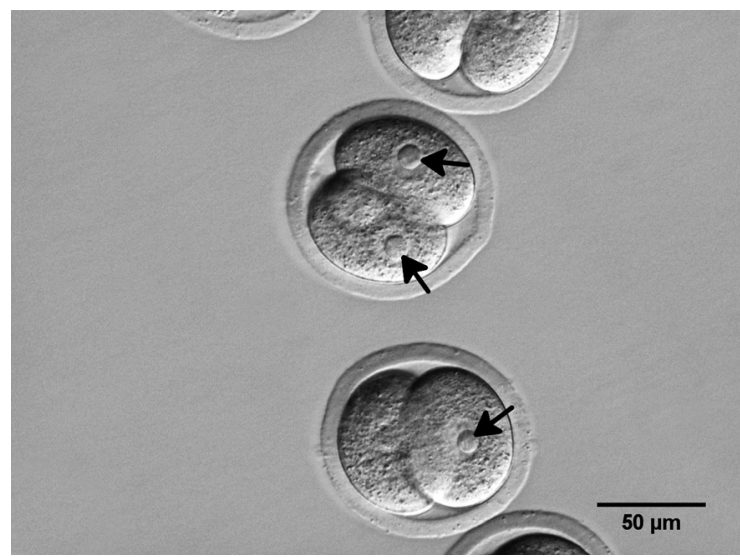

Fig. 4 (left). Injection of an extra nucleolus like body (N) into an immature mouse oocyte. The extra nucleolar material is rapidly translocated into the germinal vesicle (GV) where first very tiny NLBs are visible. These tiny NLBs gradually fuse with the original GV nucleolus and form one large NLB.

Fig. 5 (right). Development of oocytes with extranucleolar material. The oocytes with injected extra nucleolar material mature normally at rates comparable to controls. When parthenogenetically activated, the embryos develop up to the blastocyst stage. Nevertheless, certain differences can be detected when compared to non-manipulated cells. For example, in non-manipulated embryos nuclei in two-cell stage blastomeres contain several nucleoli whilst with injected extra NLB material, the nuclei contain only a single large nucleolus (arrows). Moreover, the embryos with extra nucleolar material compact earlier than controls - already in four-cell stage. 
reported to be fully fertile (Burns et al., 2003). By contrast, even a relatively mild microsurgical reduction of the NPB volume leads to severely compromised development. This is not easy to explain. It is possible that the volume must reach a certain threshold in order for the NPB to sustain its function and the correlation between the developmental competence and the NPB volume/mass might not be strictly linear. However, further experiments are needed to probe these possibilities.

\section{Conclusion}

The recently published papers strongly contradict the classical developmental biology dogma that the atypical oocyte/zygote nucleoli (NLBs/NPBs) are essential for embryonic development because they serve as a passive repository of material that is transformed into typical tripartite nucleoli as the embryo develops. Conversely, NPBs are major heterochromatin-organizing structure in oocytes and zygotes (Dang-Nguyen and Torres-Padilla, 2015) and, at least in the mouse, NPBs are indispensable only during a relatively very short time period post fertilization. Normal nucleologenesis occurs even in their absence. While we have gained a significant insight into the NLB/NPB function, many questions remain to be answered. This is especially true for human assisted reproduction where it has been reported that the distribution and number of NPBs in PNs mirrors the developmental potential of zygotes. Being strictly non-invasive, this method seems highly promising. However, there are several obstacles when address- ing the NPB functions in human embryos and thus far, we can only extrapolate their role(s) based on the mouse model. The human material is very scarce and is preferentially used for the production of test-tube babies. Zygotes are only exceptionally available for research and used for further analyses. Moreover, the use of zygotes or more advance embryos is not permitted in many countries. Thus, the most acceptable approach would be a multiparameter non-invasive evaluation of embryos. In this context, it would be especially interesting to investigate if there is a correlation between the NPB distribution and the length of the first mitotic division (Vera-Rodriguez et al., 2015). Moreover, it is evident that oocyte maturation and further embryonic development are very complex processes in which NLBs/NPBs play an essential and not negligible role (Conti and Franciosi, 2018).

\section{Challenging questions opened for future research}

Even after the most essential role of NLBs/NPBs has been clarified, many interesting questions remain. Some of them are listed below:

1/ Do NPB distribution patterns in pronuclei mirror the developmental potential of embryos in other species than humans?

2/ Are there changes in the NLB/NPB composition? Better and more detailed characterization of NSN oocyte NLBs, SN oocyte NLBs, and NPBs in zygotes and also NPBs artificially produced by the injection of Npm2 mRNA would be beneficial. Up to now, this information is only fragmentary, for example it has been reported

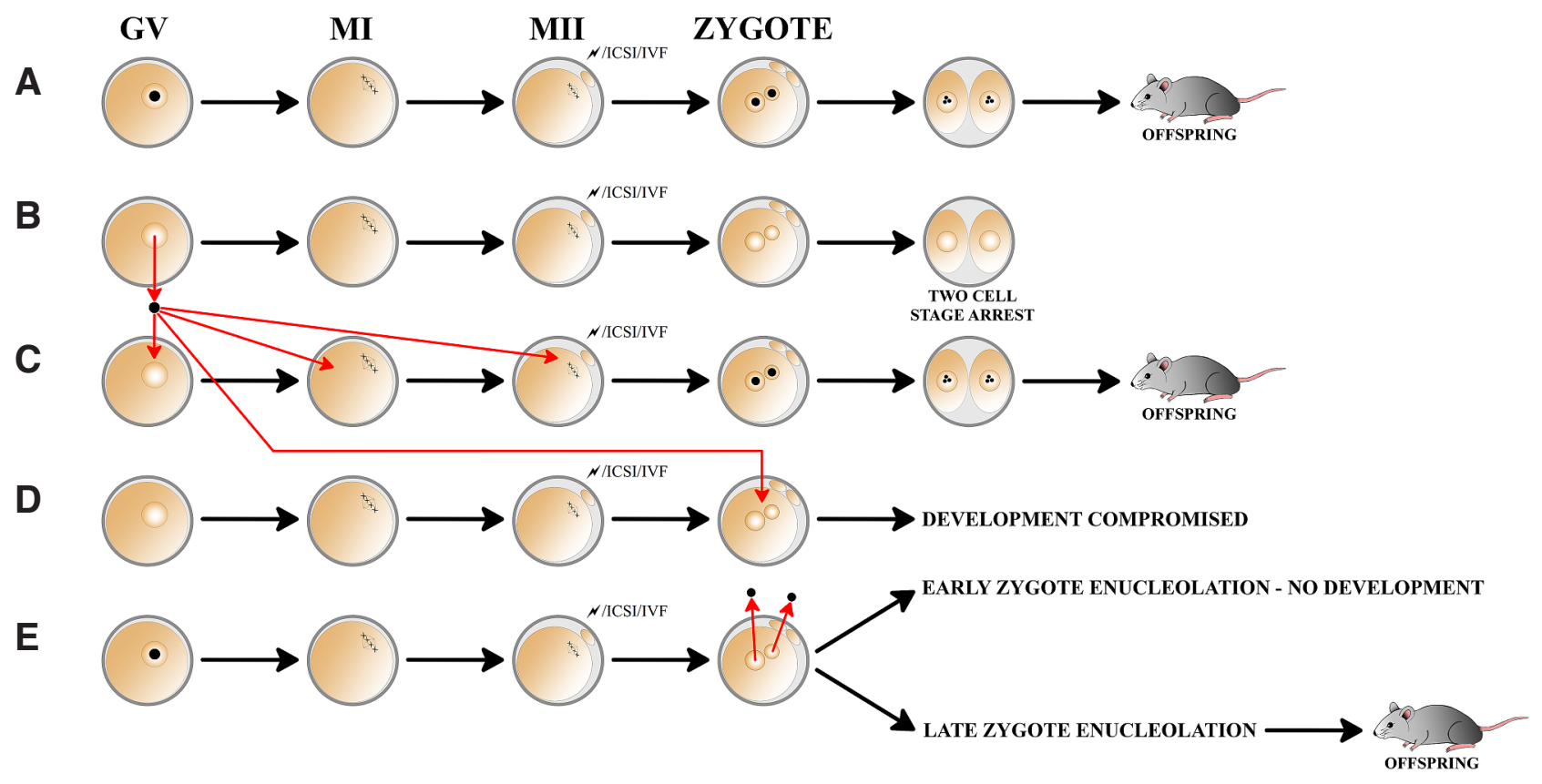

Fig. 6. Summary of mouse oocyte/zygote nucleolus manipulation experiments. (A) Maturation and development of non-manipulated oocytes. (B) Enucleolated oocytes mature normally to metaphase II (MII), but after fertilization or parthenogenetic activation the pronuclei do not contain nucleoli (NPBs). The zygotes cleave typically only once - to the two cell stage. (C) When isolated NLBs are reinjected into previously enucleolated oocytes (GV, MI, MII) and these oocytes are then fertilized, their developmental potential is fully restored with offspring born. (D) Interestingly, when NLBs are injected into zygotes originating from previously enucleolated oocytes (PNs without nucleoli), development of embryos is compromised. The injected embryos rarely reach the blastocyst stage. (E) When intracytoplasmic sperm injection (ICSI)-produced zygotes are enucleolated, their further development depends on time of enucleolation. When zygotes are enucleolated at 8 or less hours post ICSI, the embryos do not develop. On the other hand, when the enucleolation is performed at 10 or more hours post ICSI, the enucleolated embryos develop normally with offspring born. 
that NSN NLBs contain more UBF than SN NLBs. Moreover, NPBs contain less RNAs than NLBs (Lavrentyeva et al., 2017; Fulka and Langerova, 2014). It would be interesting to characterize the exact nature of these RNAs.

3/ Why are NLBs in other species than the mouse not visible in native oocytes (for example sheep, bovine)? These can be detected only by EM (electron microscopy). Is their composition different from mouse NLBs? What will happen with mouse NLBs after their injection into immature oocytes of these species. We may suppose, that at least some basic and essential molecules are present in NLBs of all mammalian species. For example, developmental potential of enucleated porcine oocytes can be rescued when mouse NLBs are injected into them (Morovic et al., 2017) and vice versa (Benc, in preparation).

4/ Which components or forces attract and organize the chromatin to/on the surface of NLBs/NPBs? Is this just NPM2?

5/ Why are NSN oocytes arrested post fertilization at the twocell stage? Does this arrest have a similar mechanism or cause as in embryos without nucleoli?

6 / Is the association of chromatin with NLBs indeed essential for oocyte maturation and especially for further development? The oocytes from $\mathrm{Npm} 2 \%$ mice as well as enucleated oocytes mature normally with no chromosomal abnormalities. Similarly, when GVs from very small oocytes (even from one day old mice) are transferred into cytoplasts obtained by enucleation of immature fully grown oocytes, their maturation is normal. Moreover, when metaphases II are then transferred into enucleated ovulated oocytes and these oocytes are then fertilized, the zygotes develop at least to the blastocysts (Bao et al., 2000). In both these cases, chromatin is not associated with the dense fibrillar NLBs as these structures are not present in nuclei/GVs. Also the exchange of whole GVs between NSN and SN oocytes showed, that whilst maturation of reconstructed oocytes in NSN nucleus $x$ $\mathrm{SN}$ cytoplasm combination is normal, further development post fertilization is compromised. The transfer of NSN MII plates into $\mathrm{SN}$ cytoplasts rescues developmental potential of reconstructed cells and they reach the blastocyst stage (Inoue et al., 2008). This rather indicates that the association of chromatin with NPBs is more essential than the association of chromatin with NLBs. Moreover, it is possible that differences in NLB composition between SN and NSN oocytes are also essential post fertilization when NLB material is relocated into PNs. Although we cannot exclude the role of some additional nuclear factors, the exchange of NLBs between NSN and SN oocytes can give us at least a partial answer. In the pig, nucleoli from growing oocytes as well as from two-cell stage blastomere nuclei rescued development of enucleolated FG (fully grown) oocytes after parthenogenetic activation (Kyogoku et al., 2010, 2012).

7/As mentioned in the text, NPBs are essential within a relatively short time interval post-fertilization. When they are removed after this interval, nucleologenesis occurs even in their absence. Up to now, only anti-B23 (nucleophosmin) antibody has been used for newly formed nucleoli detection. The use of additional antibodies (C23 - nucleolin, UBF - upstream binding factor, fibrillarin) as well as EM characterization would be interesting. Besides this, it is unclear yet how the original NPBs are eliminated when embryo develops - is autophagy at play?

8/ It has been clearly documented recently that NLBs/NPBs serve as the major heterochromatin-organizing structures in oocytes and zygotes. Which other functions, do these specific intranuclear organelles possibly have?

\section{Acknowledgements}

JFJr's lab is supported from GACR 17-08605S. P Loi's lab has received funding from the European Union's Horizon 2020 research and innovation programme under grant agreement No 734434 and No 698165.We do apologize to those authors whose work is not cited in our article. JFJr and HF dedicate this paper to Andrzej K. Tarkowski who recently passed away and whose brilliant work inspired us in many experiments.

\section{References}

AGUIRRE-LAVIN, T., ADENOT, P., BONNET-GARNIER, A., LEHMANN, G., FLEUROT, R., BOULESTEIX, C., DEBEY, P. and BEAUJEAN, N. (2012) 3D-FISH analysis of embryonic nuclei in mouse highlights several abrupt changes of nuclear organization during preimplantation development. BMC Dev Biol 12: 30.

BACHANT, J.B. and ELLEDGE, S.J. (1999) Mitotic treasures in the nucleolus. Nature 398: 757-758.

BAO, S., OBATA, Y., CARROLL, J., DOMEKI, I. and KONO, T. (2000) Epigenetic modifications necessary for normal development are established during oocyte growth in mice. Biol Reprod 62: 616-621.

BOGOLYUBOV, D.S. (2018) Karyoshere (karyosome): A peculiar structure of the oocyte nucleus. Int Rev Cell Mol Biol 337: 1-48.

BOISVERT, F.M., VAN KONINGSBRUGGEN, S., NAVASCUES, J. and LAMMOND A.I. (2007) The multifunctional nucleolus. Nat Rev Mol Cell Bio 8: 574-585.

BONNET-GARNIER, A., FEUERSTEIN, P., CHEBROUT, M., FLEUROT, R., JAN, H.U., DEBEY, P. and BEAUJEAN, N. (2012) Genome organization and epigenetic marks in mouse germinal vesicles. Int J Dev Biol 56: 877-887.

CONTI, M. and FRANCIOSI, F. (2018) Acquisition of oocyte competence to develop as an embryo: integrated nuclear and cytoplasmic events. Hum Reprod Update 24: $245-265$

BURNS, K.H., VIVEIROS, M.M., REN, Z., WANG, P., DEMAYO, F.J., FRAIL., D.E. EPPIG, J.J. and MATZUK, M.M. (2003) Roles of MPM2 in chromatin and nucleolar organization in oocytes and embryos. Science 300: 633-636.

CROZET, N. (1989) Nucleolar structure and RNA synthesis in mammalian oocytes. J. Reprod Fert Supp/ 38: 9-16.

DANG-NGUYEN, T.Q. and TORRES-PADILLA, M.E. (2015) How cells build totipotency and pluripotency: nuclear, chromatin and transcriptional architecture. Curr Opin Cell Biol 34: 9-15.

EDENS, L.J., WHITE, K.H., JEVTIC, P., LI, X. and LEVY, D. (2013) Nuclear size regulation: from single cells to development and disease. Trends Cell Biol 23: 151-159.

FULKA, JR., J., MOOR, R.M., LOI, P. and FULKA, J. (2003) Enucleolation of porcine oocytes. Theriogenology 59: 1879-1885.

FULKA, H., MRAZEK, M. and FULKA, JR., J. (2004) Nucleolar dysfunction may be associated with infertility in humans. Fertil Steril 82: 486-487.

FULKA, JR., J., LANGEROVA, A., LOI, P., MARTINKOVA, S. and FULKA, H. (2011) Transplantation of nucleoli into human zygotes: not as simple as expected? Assist Reprod Dev 28: 385-389.

FULKA, H., MARTINKOVA, S., KYOGOKU, H., LANGEROVA, A. and FULKA, JR., J. (2012) Production of giant mouse oocyte nucleoli and assessment of their protein content. J Reprod Dev 58: 371-376.

FULKA, H. and LANGEROVA, A. (2014) The maternal nucleolus plays a key role in centromere satellite maintenance during the oocyte to embryo transition. Development 141: 1694-1704.

FULKA, H., KYOGOKU, H., ZATSEPINA, O., LANGEROVA, A. and FULKA, JR., J. (2015) Can nucleoli be markers of developmental potential in human zygotes. Trends Mol Med 21: 663-672.

FULKA, H. and AOKI, F. (2016) Nucleolus precursor bodies and ribosome biogenesis in early mammalian embryos: old theories and new discoveries. Biol Reprod 94: 1-8.

GARDNER, D.K., MESEGUER, M., RUBIO, C. and TREFF, N.R. (2015) Diagnosis of human preimplantation embryo viability. Hum Reprod Update 21: 727-747.

HYTTEL, P. (2011) Electron microscopy of mammalian oocyte development, maturation and fertilization. In: Oocyte maturation and Fertilization: A long history for a short 


\section{J. Fulka et al.}

event (Eds. E. Tosti and R. Boni). Bentham Science Publishers, Shajrah, pp. 1-37.

INOUE, A., NAKAJIMA, R., NAGATA, M. and AOKI, F. (2008) Contribution of the oocyte nucleus and cytoplasm to the determination of meiotic and developmental competence in mice. Hum Reprod 23: 1377-1384.

INOUE, A., OGUSHI, S., SAITOU, M., SUZUKI, M.G. and AOKI, F. (2011) Involvement of mouse nucleoplasmin 2 in the decondensation of sperm chromatin after fertilization. Biol Reprod 85: 70-77.

INOUE, A. and AOKI, F. (2010) Role of the nucleoplasim 2 C-terminal domain in the formation of nucleolus-like bodies in mouse oocytes. Faseb J 24: 485-494.

JORDAN, E.G. (1991) Interpreting nucleolar structure: where are the transcribed genes? J Cell Sci 98: 437-442.

KYOGOKU, H., OGUSHI, S. and MIYANO, T. (2010) Nucleoli from growing oocytes support development of enucleolated full grown oocytes in the pig. Mol Reprod Dev 77: 167-173.

KYOGOKU, H., OGUSHI, S. and MIYANO, T. (2012) Nucleoli from two-cell embryos support the development of enucleated germinal vesicle oocytes in the pig. Biol Reprod 87: 1-6.

KYOGOKU, H., FULKA, JR., J., WAKAYAMA, T. and MIYANO, T. (2014) De novo formation of nucleoli in developing mouse embryos originating from enucleolated zygotes. Development 141: 2255-2259.

KYOGOKU, H., WAKAYAMA, T., KITAJIMA, T.S. and MIYANO, T. (2018) Single nucleolus precursor body formation in the pronucleus of mouse zygotes and SCNT embryos. PLOS ONE 13: e0202663.

MOROVIC, M., STREJCEK, F., NAKAGAWA, S., DESHMUKH, R.S., MURIN, M., BENC, M., FULKA, H., KYOGOKU, H., PENDOWSKI, L., FULKA, JR., J. and LAURINCIK, J. (2017) Mouse oocytes nucleoli rescue embryonic development of porcine enucleolated oocytes. Zygote 25: 675-685

LAVRENTYEVA, E.A., SHISHOVA, K., KAGARLITSKY, G. and ZATSEPINA, O. (2017) Localization of RNAs and proteins in nucleolar precursor bodies of early mouse embryos. Reprod Fert Develop 29: 509-520.

OGUSHI, S., PALMIERI, C., FULKA, H., SAITOU, M., MIYANO, T. and FULKA, JR., $\mathrm{J}$. The maternal nucleolus is essential for embryonic development in mammals.
Science 319: 613-616

OGUSHI, S. and SAITOU, M. (2010) The nucleolus in the mouse oocyte is required for the early step of both female and male pronucleus organization. J Reprod Dev 56: 495-501.

OGUSHI, S., YAMAGATA, K., OBUSE, C., FURUTA, K., WAKAYAMA, T., MATZUK, M.M. and SAITOU, M. (2017) Reconstitution of the oocyte nucleolus in mice trough a single nucleolar protein. J Cell Sci 130: 2416-2429.

PAPALE, L., FIORENTINO, A., MONTAG, M. and TOMASI, G. (2012) The zygote. Hum Reprod 51: 122-149.

POCHUKALINA, G.N., ILICHEVA, N.V., PODGORNAYA, O.I. and VORONIN, A.P. (2016) Nucleolus-like body of the mouse contains lamin A and $B$ and TRF2 but not actin and topo II. Mol Cytogenet 9: 50.

ROMANOVA, L.G., ANGER, M., ZATSEPINA, O.V. and SCHULTZ, R.M. (2006) Implication of nucleolar protein SURF6 in ribosome biogenesis and preimplantation mouse development. Biol Reprod 75: 690-696.

SHISHOVA, K.V., LAVRENTYEVA, E.A., DOBRUCKI, J.W. and ZATSEPINA, O.V. (2015) Nucleolus-like bodies of fully-grown mouse oocytes contain key nucleolar proteins but are impoverished for rRNA. Dev Biol 397: 267-281

TESARIK, J. and GRECO, E. (1999) The probability of abnormal preimplantation development can be predicted by a single static observation on pronuclear stage morphology. Hum Reprod 14: 1318-1323.

VERA-RODRIGUEZ, M., CHAVEZ, S.L., RUBIO, C., REIJO PERA, R.A., SIMON C. (2015) Prediction model for aneuploidy in early human embryos development revealed by single-cell analysis. Nat Commun 6: 7601.

VOGT, E.J., MEGLICKI, M., HARTUNG, K.I., BORSUK, E. and BEHR, R. Importance of the pluripotency factor LIN28 in the mammalian nucleolus during early embryonic development. Development 139: 4514-4523.

ZINK, D., FISHER, A.H. and NICKERSON, J.A. (2004) Nuclear structure in cancer cells. Nat Rev Cancer 4: 677-687.

ZUCCOTTI, M., MERICO, V., CECCONI, S., REDI, C.A. and GARAGNA, S. (2011) What does it take to make a developmentally competent mammalian egg? Hum Reprod Update 17: 525-540. 


\section{Further Related Reading, published previously in the Int. J. Dev. Biol.}

Differential regulation of cumulus cell transcription during oocyte maturation in vivo and in vitro

Giovanni Coticchio, Libby Ophir, Yuval Yung, Micha Baum, Mariabeatrice Dal Canto, Mario Mignini-Renzini, Fausta Brambillasca, Rubens Fadini and Ariel Hourvitz

Int. J. Dev. Biol. (2017) 61: 433-437

https://doi.org/10.1387/ijdb.160364gc

All in one - integrating cell polarity, meiosis, mitosis and mechanical forces in early oocyte differentiation in vertebrates Yaniv M. Elkouby

Int. J. Dev. Biol. (2017) 61: 179-193

https://doi.org/10.1387/ijdb.170030ye

The effect of amniotic membrane stem cells as donor nucleus on gene expression in reconstructed bovine oocytes Hassan Nazari, Abolfazl Shirazi, Naser Shams-Esfandabadi, Azita Afzali and Ebrahim Ahmadi Int. J. Dev. Biol. (2016) 60: 95-102

https://doi.org/10.1387/ijdb.160010hn

Reprogramming of somatic cells and nuclei by Xenopus oocyte and egg extracts Alexander A. Tokmakov, Tetsushi Iwasaki, Ken-Ichi Sato and Shinji Kamada Int. J. Dev. Biol. (2016) 60: 289-296

https://doi.org/10.1387/ijdb.160163at

\section{Human oocyte maturation in vitro}

Giovanni Coticchio, Mariabeatrice Dal-Canto, Maria-Cristina Guglielmo, Mario Mignini-Renzini and Rubens Fadini

Int. J. Dev. Biol. (2012) 56: 909-918

https://doi.org/10.1387/ijdb.120135gv

Strategies to support human oocyte development in vitro

Evelyn E. Telfer and Marie McLaughlin

Int. J. Dev. Biol. (2012) 56: 901-907

https://doi.org/10.1387/ijdb.130001et

Spatial arrangement of intra-nucleolar rDNA chromatin in amplified Xenopus oocyte nucleoli: structural changes precede the onset of rDNA transcription H Spring, B Meissneer, R Fischer, D Mouzaki and M F Trendelenburg Int. J. Dev. Biol. (1996) 40: 263-272

http://www.intjdevbiol.com/web/paper/8735937
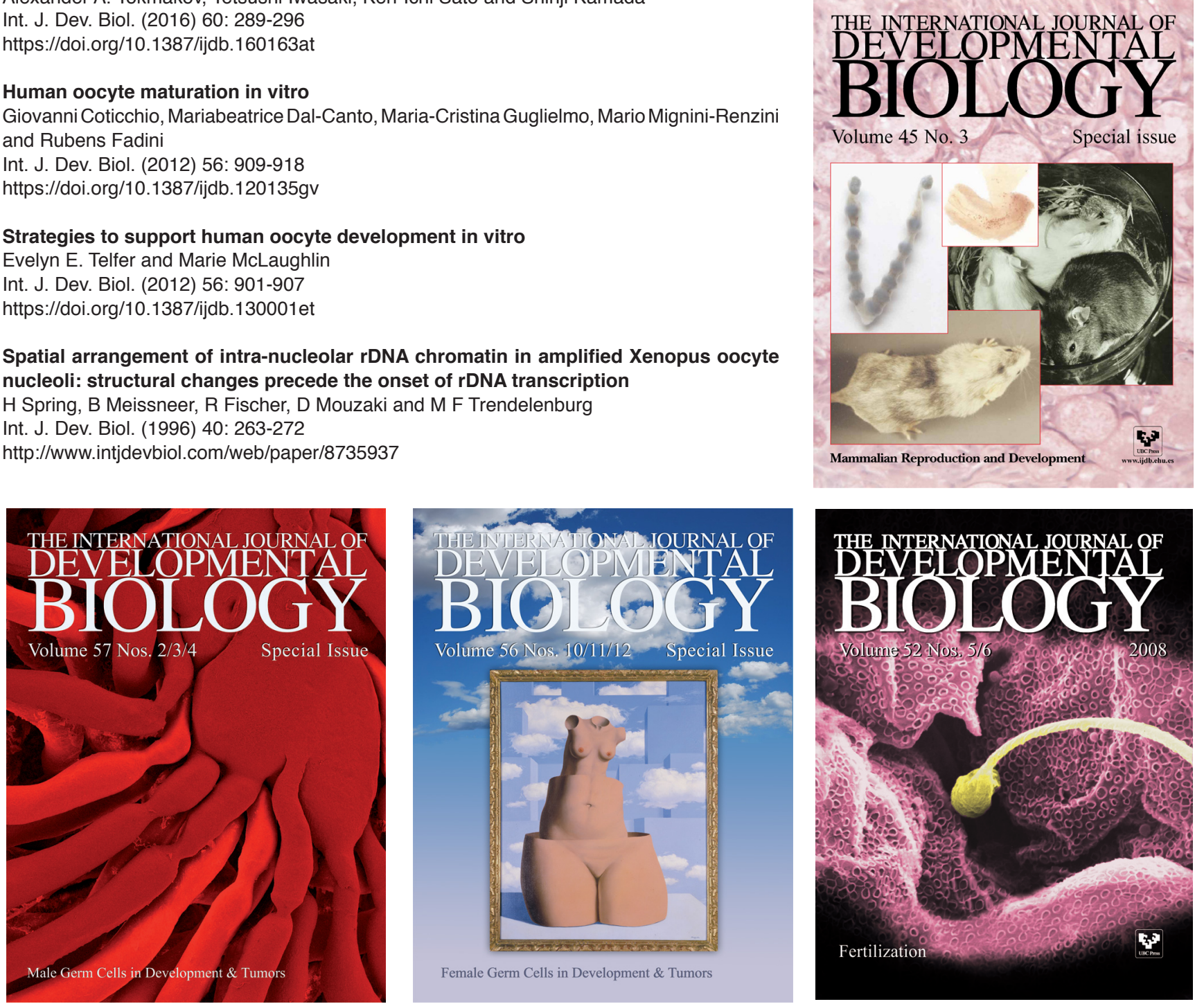